Fakten und Statistiken 2005

\title{
1. Aussenpolitik
}

\section{Catherine Schümperli Younossian}

\section{(2) OpenEdition}

\section{Journals}

Electronic version

URL: http://journals.openedition.org/sjep/177

DOI: $10.4000 /$ sjep. 177

ISSN: 1663-9677

\section{Publisher}

Institut de hautes études internationales et du développement

\section{Printed version}

Date of publication: 1 avril 2005

Number of pages: 3-14

ISBN: 2-88247-058-4

ISSN: $1660-5926$

\section{Electronic reference}

Catherine Schümperli Younossian, «1. Aussenpolitik », Schweizerisches Jahrbuch für Entwicklungspolitik [Online], 24-1 | 2005, Online erschienen am: 19 April 2010, abgerufen am 08 September 2020. URL : http://journals.openedition.org/sjep/177 ; DOI : https://doi.org/10.4000/sjep. 177

(c) The Graduate Institute 


\section{Aussenpolitik*}

D IE HAUPTZÜGE der schweizerischen Aussenpolitik sind in der Bundesverfassung verankert und werden vom Bundesrat in seinen Dokumenten über die strategische Ausrichtung seiner Politik präzisiert, z.B. in Berichten über die Aussenpolitik oder über die Legislaturplanung. Für die Umsetzung dieser Leitlinien ist das Eidgenössische Departement für auswärtige Angelegenheiten (EDA) zuständig.

Die Schweiz ist seit September 2002 Mitglied der Vereinten Nationen und beteiligt sich aktiv an deren Arbeiten, namentlich an dem von Generalsekretär Kofi Annan eingeleiteten Reformprozess. Rund 30 Schweizer Persönlichkeiten sind in den leitenden Organen der Vereinten Nationen oder deren Sonderorganisationen tätig.

Ein Meilenstein des Jahres 2004 war der Abschluss der Bilateralen Verträge II mit der Europäischen Union, die sich zum Teil auf heikle Themenbereiche wie Asylwesen, Rechtshilfe oder Bankgeheimnis beziehen. Ferner hat die Schweiz zugesagt, sich am Kohäsionsfonds der erweiterten EU zu beteiligen.

Die Beziehungen zwischen der Schweiz und dem südafrikanischen Apartheidregime werden in einem neu erschienenen Bericht ausgeleuchtet. Gegenstand dieser Untersuchung bilden insbesondere die Beziehungen des Schweizer Nachrichtendienstes zu südafrikanischen Stellen.

\subsection{Grundzüge der schweizerischen Aussenpolitik}

Die Umsetzung der Aussenpolitik der Schweiz ist Aufgabe des Eidgenössischen Departementes für auswärtige Angelegenheiten. Die am 1. April 2000 in Kraft getretene Bundesverfassung nennt fünf Schwerpunkte für die auswärtigen Beziehungen: „,Der Bund setzt sich ein für die Wahrung der Unabhängigkeit der Schweiz und für ihre Wohlfahrt; er trägt namentlich bei zur Linderung von Not und Armut in der Welt, zur Achtung der Menschenrechte und zur Förderung der Demokratie, zu einem friedlichen Zusammenleben der Völker sowie zur Erhaltung der natürlichen Lebensgrundlagen“ (Art. 54 Abs. 2). (Siehe nachstehenden Rahmentext „Die fünf Schwerpunkte der schweizerischen Aussenpolitik“).

\subsubsection{Legislaturplanung 2003-2007}

Ende Februar 2004 verabschiedete der Bundesrat die Legislaturplanung für die Jahre 2003 bis 2007'. Der Bericht vermittelt eine Gesamtübersicht über die Strategie des Bundesrates zur Bewältigung der wichtigsten Herausforderungen, mit denen die Schweiz in den kommenden vier Jahren konfrontiert ist.

In seinem Bericht nannte der Bundesrat drei Leitlinien für die Zukunft:

1. Den Wohlstand vermehren und die Nachhaltigkeit sichern.

2. Die demografischen Herausforderungen bewältigen.

* Von Catherine Schümperli Younossian, Forschungsbeauftragte am iuéd.

1 Bericht über die Legislaturplanung 2003-2007 vom 25. Februar 2004 (BB1 2004 1149). 
3. Die Stellung der Schweiz in der Welt festigen.

Die Legislaturfinanzplanung ist ebenfalls Bestandteil des Berichtes.

Für die auswärtigen Beziehungen ist insbesondere die dritte Leitlinie massgebend. In diesem Zusammenhang wird festgehalten, dass die Schweiz ihre Verantwortung innerhalb der Staatengemeinschaft durch eine verstärkte Präsenz auf der internationalen Bühne und durch eine intensivierte Zusammenarbeit wahrnehmen will. Der Bundesrat bekräftigt diese Absicht wie folgt: „Wir wollen zu Frieden, Achtung der Menschenrechte, Wohlstand, Verminderung der Armut und zur Erhaltung der Umwelt beitragen. Das macht uns zu vertrauenswürdigen Partnern und festigt unsere Position in der Welt. Gleichzeitig eröffnen wir uns so den notwendigen Spielraum, um die Interessen unseres Landes wirksam und glaubwürdig vertreten zu können. Glaubwürdig können wir allerdings nur sein, wenn es uns gelingt, unsere wirtschaftlichen Interessen mit unserem Bestreben um eine gerechtere, friedlichere Welt in Einklang zu bringen. " ${ }^{2}$

Konkretisiert wird diese dritte Leitlinie durch drei Zielsetzungen mit entsprechenden Massnahmen, nämlich die Klärung und Vertiefung der Beziehungen zur Europäischen Union (Konsolidierung und Erweiterung des bilateralen Rahmens und Evaluation der Auswirkungen eines Beitritts zur EU), die Wahrnehmung der internationalen Verantwortung (Umsetzung der Prioritäten der schweizerischen Aussenpolitik und Wahrung der Chancen für schweizerische Exporte) sowie die Gewährleistung der Sicherheit (Umsetzung der neuen Sicherheitspolitik und Optimierung der internationalen Zusammenarbeit).

Im Gegensatz zu früher muss die Legislaturplanung seit dem In-Kraft-Treten des neuen Parlamentsgesetzes am 1. Dezember 2003 den Eidgenössischen Räten vorgelegt werden, welche anschliessend die übergeordneten Ziele der Bundespolitik in einem einfachen Bundesbeschluss verabschieden. Diese neue Rechtslage stärkt die Bedeutung und den rechtlichen Status der Legislaturplanung, die in der Vergangenheit vom Parlament lediglich zur Kenntnis genommen wurde.

Die Eidgenössischen Räte befassten sich in der Sommersession 2004 mit dem Geschäft ${ }^{3}$. Die Planung konnte nach den lebhaften Beratungen in der Sommersession 2004 nicht verabschiedet werden, da alle Parteien scharfe Kritik daran geäussert hatten. Trotz aller Aufrufe, die Divergenzen zwischen den Parteien zu überwinden und sich im Interesse des Landes auf allgemeine Leitlinien für die strategische Ausrichtung der eidgenössischen Politik zu einigen, nutzten die Parteien die Debatte, um ihre eigenen politischen Forderungen geltend zu machen.

Der Nationalrat befasste sich als Erstrat mit der Legislaturplanung, die einen ausgewachsenen ideologischen Glaubenskrieg zwischen der Linken (angeführt von den Grünen, die einen ökologischen Rahmen forderten) und der Rechten (allen voran die SVP, die die gesamte Legislaturplanung der Schuldenbremse unterstellen wollte) auslöste. Die SP drohte mit Widerstand in der Schlussabstimmung, falls sämtliche sozialen Anliegen abgelehnt und gewisse Vorstösse der Rechten in das Programm aufgenommen würden. Die CVP nahm eine gemässigte Position ein, während die gespaltene FDP mehrheitlich die Argumente der Rechten unterstützte ${ }^{4}$. Im Anschluss an die Differenzbereinigung zwi-

\footnotetext{
Ibid., S. 1179.

Bundesversammlung, Amtliches Bulletin, Sommersession 2004, <www.parlament.ch>.

„Pénible exercice sur les projets du Conseil fédéral“, Le Courrier, 4. Juni 2004; „,Le Programme de législature provoque un affrontement idéologique au Parlement“, Le Temps, 4. Juni 2004.
} 
schen den Kammern wurde die Legislaturplanung in der Schlussabstimmung vom Nationalrat abgelehnt, während der Ständerat zustimmte. Trotz der Ablehnung im Parlament wird die Schweiz über politische Leitlinien verfügen, denn der Bundesrat ist laut Verfassung beauftragt, die Ziele und Mittel seiner Politik zu definieren.

\section{Die fünf Schwerpunkte der schweizerischen Aussenpolitik}

In seinem im November 2000 verabschiedeten Aussenpolitischen Bericht 2000ª präsentierte der Bundesrat die Leitlinien und Schwerpunkte der Aussenpolitik für die nächsten zehn Jahre, die sich in fünf operativen Zielen zusammenfassen lassen. Jedes Jahr zieht das Schweizerische Jahrbuch für Entwicklungspolitik Bilanz der Umsetzung der schweizerischen Aussenpolitik in diesen fünf Schwerpunktbereichen.

口 Intensivierung der Friedensförderung, namentlich in Bezug auf die Konfliktverhütung und den Wiederaufbau, sowie Stärkung der Offenheit zum Dialog und der Achtung kultureller Vielfalt

[Dahrbuch 2005, Nr. 1, Kapitel 11, „Friedens- und Sicherheitspolitik”.

๑ Weiterführung der humanitären Politik der Schweiz, weltweiter Einsatz für Menschenrechte, Demokratie und Rechtsstaat

$\mathbb{1}$ Jahrbuch 2005, Nr. 1, Kapitel 4, „Humanitäre Hilfe“, und 9 "Menschliche Entwicklung und Menschenrechte".

口 Wahrung der wirtschaftlichen Interessen der Schweiz durch die aussenpolitische Abstützung des Standorts Schweiz und die Schaffung bestmöglicher Rahmenbedingungen im Inland

[Dahrbuch 2005, Nr. 1, Kapitel 5, „Aussenwirtschaftspolitik“.

ـ Ausbau der Entwicklungszusammenarbeit, insbesondere im Hinblick auf die Armutsbekämpfung

$\mathbb{Q}$ Jahrbuch 2005, Nr. 1, Kapitel 2, „Zusammenarbeit mit den Entwicklungsländern“ und 3 „Zusammenarbeit mit Osteuropa und der GUS".

- Mitarbeit bei der Errichtung eines internationalen rechtlichen Rahmenwerks im Umweltbereich

Jahrbuch 2005, Nr. 1, Kapitel 10, „Umwelt und Entwicklung”.

a Aussenpolitischer Bericht 2000 - Präsenz und Kooperation: Interessenwahrung in einer zusammenwachsenden Welt, 15. November 2000 (BBI 2001 261).

\subsection{Das Eidgenössische Departement für auswärtige Angelegenheiten im Jahr 2004}

\subsubsection{Jahresmedienkonferenz des EDA}

Im Rahmen der jährlichen Medienkonferenz des EDA im April $2004^{5}$ zog die Vorsteherin des Eidgenössischen Departementes für auswärtige Angelegenheiten, Micheline Calmy-Rey, die Bilanz der Arbeit ihres Departementes. In ihrer Ansprache bekräftigte Bundesrätin Calmy-Rey die Angemessenheit einer sichtbaren und aktiven Aussenpolitik: ,Aussenpolitik kann nicht ausschliesslich als Handelspolitik verstanden werden. Ob wir unsere Sicherheit und unseren Wohlstand erfolgreich verteidigen können, hängt auch davon ab, wie stark wir auf dem internationalen Parkett präsent sind und geachtet werden, und zwar sowohl auf bilateraler als auch auf multilateraler Ebene. ${ }^{" 6}$ 


\section{Das EDA 2004 : Fakten und Zahlen}

Mitarbeitende des EDA

Bei der Umsetzung der schweizerischen Aussenpolitik kann sich das EDA auf rund 3200 Mitarbeitende verlassen, die sich wie folgt auf die einzelnen Dienststellen verteilen:

- allgemeine Dienste: 700 Personen;

- diplomatische und konsularische Auslandsvertretungen der Schweiz: 2000 Personen;

- Direktion für Entwicklung und Zusammenarbeit (DEZA): 550 Personen.

\section{Schweizerische Vertretungen im Ausland}

Mehr als 300 Aussenvertretungen stellen die Pflege der diplomatischen Beziehungen zu anderen Ländern und die Teilnahme an internationalen Konferenzen sicher. 93 Botschaften, 44 Generalkonsulate und 12 Ständige Vertretungen bilden ein Netz von rund 150 offiziellen Vertretungen, das durch ungefähr 50 Kooperationsbüros ergänzt wird.

Finanzielle Ressourcen

2003 belief sich das Budget des EDA auf insgesamt 1875 Millionen Franken, wovon 1200 Millionen von der Direktion für Entwicklung und Zusammenarbeit (DEZA) verwaltet wurden. Die Aufwendungen des EDA für die Pflege der Aussenbeziehungen entsprechen 1 Prozent der Gesamtausgaben des Bundes.

Rücktritt im EDA

Nach 28 Jahren im Dienste des Bundes, davon knapp sechs Jahre als Staatssekretär im EDA (1999 bis Mitte Februar 2005), tritt Franz von Däniken im Februar 2005 von seinem Amt zurück. Sein Nachfolger ist Michael Ambühl, ehemaliger Chef des Integrationsbüros EDA/EVD in Bern.

Quellen: Bundeskanzlei, Der Bund kurz erklärt, Bern, März 2004; Presse- und Informationsdienst EDA.

\subsubsection{Schaffung eines Kompetenzzentrums für Kulturaussenpolitik}

$\mathrm{Zu}$ den wichtigsten Themen der Jahresmedienkonferenz ${ }^{7}$ gehörte insbesondere auch die Schaffung eines Kompetenzzentrums für Kulturaussenpolitik, welche das kulturelle Schaffen der Schweiz im Ausland fördern soll. Angesichts der zahlreichen Akteure im Kulturbereich und der Notwendigkeit, die bisher fehlenden Synergien zu entwickeln, soll das neue Kompetenzzentrum die ehemalige Sektion „Kultur und UNESCO“ ersetzen. Das Zentrum soll vier Ziele erfüllen: 1) Umsetzung der Kulturaussenpolitik des Departementes, 2) Durchführung von Kulturprojekten in Zusammenarbeit mit Schweizer Vertretungen im Ausland, 3) Vertretung der Schweizer Politik in der UNESCO sowie 4) Koordination der Kulturaussenpolitik der Schweiz mit den im Ausland tätigen schweizerischen Kulturinstitutionen. Das neue Zentrum versteht sich als Koordinationsplattform für Tätigkeiten auf diesem Gebiet. Zu diesem Zweck wurde eine Arbeitsgruppe mit Vertreterinnen und Vertretern der DEZA, von Pro Helvetia, von Präsenz Schweiz und des Bundesamtes für Kultur eingesetzt ${ }^{8}$.

Nicolas Bideau, der Vorsteher des neuen Zentrums, präzisierte in einem Interview dessen Ziele und die Komplementarität des Zentrums mit Pro Helvetia: Grundlage für die Förderung von Projekten durch Pro Helvetia sei primär deren

7 Folgende weitere Themen wurden erörtert: Schweizerische Menschenrechtspolitik, internationale Zusammenarbeit der Schweiz, Tätigkeit der Schweiz im Rahmen der UNO, Engagement der Schweiz in der zivilen Friedensförderung sowie Genfer Initiative. Sie werden in den verschiedenen Kapiteln des vorliegenden Jahrbuchs diskutiert.

8 EDA, „Kompetenzzentrum für Kulturaussenpolitik“, Jahresmedienkonferenz, 2004, Pressedossier, 22. April 2004. 
künstlerische Qualität. Das Zentrum achte zwar ebenfalls auf die Form, im Vordergrund stehe aber, dass die Projekte eine Botschaft vermitteln, welche den Anliegen der schweizerischen Aussenpolitik entsprechen: Achtung der Menschenrechte, Förderung des Friedens und Entwicklung. Pro Helvetia und das Zentrum seien auf unterschiedlichen „Märkten“ tätig: die Kulturstiftung primär in den Industrieländern, das Zentrum hingegen in den Ländern des Südens. ${ }^{9}$

\subsubsection{Botschafterkonferenz 2004}

Vom 23. bis zum 26. August 2004 fand in Bern die jährliche Botschafterkonferenz statt. Bereits zum zweiten Mal in Folge nahmen auch die Leiterinnen und Leiter der Koordinationsbüros der DEZA daran teil. Damit sollten der einheitliche Auftritt der Schweiz im Ausland gestärkt und die Synergien zwischen den Direktionen des EDA besser genutzt werden. Mit einem Tag „Die Welt im Jahr 2015“ legte die Konferenz den Schwerpunkt auf die Perspektiven künftiger globaler Entwicklungen (Umwelt, Wirtschaft, Demografie, neue Technologien, Ideologien und Religionen), welche die internationalen Beziehungen und die Aussenpolitik der Schweiz beeinflussen ${ }^{10}$.

In ihrer Eröffnungsansprache ${ }^{11}$ erinnerte die Departementsvorsteherin daran, dass die Beziehungen zur Europäischen Union auch in Zukunft eine Priorität der schweizerischen Aussenpolitik bilden werden, dass aber die Sicherheit und das Wohl der Schweizerinnen und Schweizer nicht allein durch den Abschluss bilateraler Verträge und nicht einmal durch den Beitritt der Schweiz zur Europäischen Union gewährleistet werden könnten. Ferner sprach sie sich für eine neutrale, aber aktivere, auf das Recht und auf die Reform der internationalen Organisationen abgestützte Aussenpolitik aus. Micheline Calmy-Rey erklärte, die Bemühungen der Schweiz für die Verwirklichung der Millenniumsziele seien ungenügend, und wies darauf hin, dass die Entwicklungszusammenarbeit und die zivile Friedensförderung vom Entlastungsprogramm für den Bundeshaushalt besonders stark betroffen seien. Zudem werde sich der Spardruck auch auf das Vertretungsnetz im Ausland auswirken. Ob Vertretungen geschlossen werden müssten, werde noch geprüft.

\subsubsection{Von der Schweiz 2003 abgeschlossene internationale Abkommen}

Im Mai 2004 verabschiedete der Bundesrat den Bericht über die im Jahr 2003 abgeschlossenen internationalen Verträge. Der im Bundesblatt ${ }^{12}$ veröffentlichte Bericht nennt insgesamt 233 internationale Vereinbarungen, wovon 169 von der DEZA abgeschlossen wurden. Zu jedem von der Schweiz rechtskräftig abgeschlossenen internationale Vertrag, für den keine Zustimmung des Parlamentes erforderlich war, bietet der Bericht eine kurze Zusammenfassung über den Inhalt, die Beweggründe, die voraussichtlichen Kosten, die Rechtsgrundlage für den Abschluss sowie die Modalitäten des In-Kraft-Tretens und der Kündigung ${ }^{13}$.

\footnotetext{
„L'art au service de la politique étrangère“, Le Courrier, 5. August 2004.

EDA, Botschafterkonferenz 2004, Pressemitteilung, 19. August 2004.

Botschafterkonferenz 2004. Ansprache von Bundesrätin Micheline Calmy-Rey.

12 Bericht über die im Jahr 2003 abgeschlossenen internationalen Verträge vom 18. Mai 2004 (BB1 2004 3319).

13 EDA, Bericht des Bundesrats über die im Jahr 2003 abgeschlossenen Verträge, Pressemitteilung, 19. Mai 2004.
} 
Seit dem Beitritt der Schweiz zu den Vereinten Nationen erstattet der Bundesrat dem Parlament jährlich Bericht über die schweizerischen Beziehungen zur UNO und zu den internationalen Organisationen mit Sitz in der Schweiz. Die Hauptanliegen der Eidgenossenschaft innerhalb der UNO sind die Reformbestrebungen, welche auf eine Stärkung der Rolle der Vereinten Nationen abzielen, das Engagement zu Gunsten der nachhaltigen Entwicklung, die Umsetzung der Millenniumsdeklaration sowie die Förderung der menschlichen Sicherheit.

\subsubsection{Bericht 2004 über die Beziehungen zur UNO und zu den internationalen Organisationen mit Sitz in der Schweiz vom 7. Juni 2004}

Auf Wunsch des Parlaments widmet dieser zweite Bericht der Reform der Vereinten Nationen ein eigenes Kapitel. In seiner Einführung geht der Bundesrat ausführlich auf die Konsequenzen der Irak-Krise für die UNO ein und unterstreicht, dass die Diskussion über die erforderlichen Reformen damit neue Impulse erhalten hat. Ferner wird die Sitzstaatpolitik der Schweiz erläutert. Der Bericht deckt den Zeitraum zwischen März 2003 und März $2004 \mathrm{ab}^{14}$.

Anlässlich der Eröffnung der 58. Session der Generalversammlung im September 2003 setzte der Generalsekretär ein mit internationalen Persönlichkeiten besetztes Gremium ein, das den Auftrag erhielt, die derzeitigen Bedrohungen zu analysieren und Empfehlungen zu Veränderungen auszuarbeiten, die notwendig sind, um die Wirksamkeit eines kollektiven Vorgehens sicherzustellen. Als konkreten Beitrag zur Tätigkeit dieses Gremiums befasste sich die Schweiz mit der Frage der Selbstverteidigung. In diesem Zusammenhang wurde im März 2004 in Genf unter dem Vorsitz des Rechtsberaters des EDA Nicolas Michel ein Workshop durchgeführt, an dem rund zwanzig international anerkannte Sachverständige teilnahmen ${ }^{15}$.

\section{$\square$ Reformen in den Vereinten Nationen}

Unmittelbar nach seinem Amtsantritt als Generalsekretär der Vereinten Nationen hatte Kofi Annan seine Absicht bekundet, eine Reihe von Reformen innerhalb der Organisation durchzuführen. Im Herbst 1997 wurde ein erstes Reformpaket präsentiert, dessen Umsetzung grösstenteils abgeschlossen ist (Sanierung des Haushalts, Steigerung der Effizienz und Bezeichnung von vier strategischen Prioritäten, nämlich Friedensförderung, wirtschaftliche und soziale Entwicklung, humanitäre Hilfe und Menschenrechte). Im Herbst 2002 legte der Generalsekretär ein zweites Bündel von Massnahmen vor, dessen Diskussion in der Generalversammlung noch nicht abgeschlossen ist. Die bisher eingeleiteten Reformen betreffen primär die internen Arbeitsweisen der Organisation, aber weder die grundlegenden Funktionsweisen der Organe der UNO noch eine zumindest teilweise - Revision der Charta der Vereinten Nationen. Dabei gilt es

14 EDA, Die Schweiz und die UNO. Bericht des Bundesrats 2004, Bern, 2004. Ebenfalls erschienen im Bundesblatt: Bericht 2004 über die Beziehungen zur UNO und zu den internationalen Organisationen mit Sitz in der Schweiz vom 7. Juni 2004 (BB1 2004 3885).

15 EDA, „UNO-Reform: Neuer Anlauf im Zeichen neuer Bedrohungen“, Schweiz Global, Nr. 3, 2004, S. 11. 
zu bedenken, dass der Generalsekretär gewisse interne Reformen anordnen kann, während für grundlegende Neuerungen ein Beschluss der Mitgliedsstaaten erforderlich ist. Der Bericht weist darauf hin, dass ein Grossteil der bisher mit Erfolg durchgeführten Reformen zur ersten Kategorie gehört, während weiter gehende Reformen, die von den Mitgliedern beschlossen werden müssen, bislang auf sich warten lassen ${ }^{16}$.

\section{$\square$ Institutionelle Reformen}

- Revitalisierung der Generalversammlung. Als einziges Organ, in dem sämtliche Mitgliedsstaaten vertreten sind, spielt die Generalversammlung innerhalb der UNO eine zentrale Rolle. Zudem ist sie das einzige Organ, das strikt egalitär funktioniert, entsprechend dem Grundsatz „Ein Staat, eine Stimme“. In den vergangenen Jahrzehnten haben jedoch die stark befrachteten Tagesordnungen, schwerfällige Verfahren und langwierige und häufig sich wiederholende Debatten die Autorität und die Rolle der Generalversammlung geschwächt. Die Reformvorschläge zielen auf eine verbesserte Zusammenarbeit zwischen den einzelnen Organen und den Präsidenten der verschiedenen Gremien ab. Die Schweiz unterstützt die Revitalisierung der Generalversammlung, formulierte jedoch drei Bedingungen: Die vorgeschlagenen Massnahmen dürfen nicht mit zusätzlichen Kosten verbunden sein, die Umgestaltung des Zeitplans darf nicht zu einer Zunahme oder Verlängerung der Tagungen führen, und die Massnahmen zur Revitalisierung müssen mit der Reform vereinbar sein, die im Bereich des Budgetzyklus der UNO eingeleitet worden ist ${ }^{17}$.

- Reform des Sicherheitsrates. Die Frage nach einer Reform des Sicherheitsrates wird innerhalb der UNO seit Jahren debattiert. Die Vorschläge, die auf eine veränderte Zusammensetzung des Sicherheitsrates und auf eine Neuregelung des Vetorechtes abzielen, liegen derzeit auf Eis. Die Schweiz befürwortet eine Erweiterung des Sicherheitsrates, da dies eine bessere Vertretung der verschiedenen Regionen der Welt gewährleisten würde, zeigt sich jedoch in Bezug auf eine Erhöhung der Anzahl Mitglieder zurückhaltend. Eine Abschaffung des Vetorechts hält die Schweiz allerdings für illusorisch; daher setzt sie sich daher verstärkt dafür ein, die Nutzung dieses besonderen Vorrechts einzuschränken. Die bereits unternommenen Bemühungen haben die Arbeitsmethoden des Sicherheitsrates in den vergangenen Jahren verbessert und mehr Transparenz sowie mehr Mitwirkungsmöglichkeiten für Nichtmitglieder gewährleistet. Zudem hat der Sicherheitsrat neue operationelle Instrumente entwickelt, mit denen sich die Umsetzung der Beschlüsse besser kontrollieren lassen. Im Hinblick auf die Sanktionen schliesslich wurden unter anderem dank dem Beitrag der Schweiz erhebliche Fortschritte erzielt: Mit gezielten Sanktionen wird gegen die Regierenden von Ländern vorgegangen, die gegen das Völkerrecht verstossen haben (indem diese an Auslandsreisen gehindert oder ihre Konten gesperrt werden $)^{18}$.

16 EDA, Die Schweiz und die UNO, op. cit., S. 16.

17 Ibid., S. 17.

18 Ibid., S. 18-19. Die Wortmeldung des Vertreters der Schweiz bei den Vereinten Nationen zur Frage der Reform des Sicherheitsrates wurde am 18. Oktober 2004 von der Westschweizer Tageszeitung Le Temps gedruckt. 
- Stärkung der UNO im wirtschaftlichen und sozialen Bereich. Die Stärkung der Vereinten Nationen im wirtschaftlichen und sozialen Bereich kann auf mehrere Arten erreicht werden: durch die Aufwertung und Reform des Wirtschafts- und Sozialrates (ECOSOC) und seiner Fachkommissionen, durch die Stärkung des Departements Wirtschaftliche und Soziale Angelegenheiten im Sekretariat der Vereinten Nationen und durch die (bereits eingeleitete) Neugestaltung der Arbeitsprogramme der Fachkommissionen der UNO-Generalversammlung. Die Schweiz befürwortet und unterstützt diese Stärkung, weil diese es ermöglichen wird, die Zusammenhänge zwischen internationaler Stabilität und wirtschaftlicher Entwicklung stärker zu berücksichtigen. Ferner ist die Schweiz der Auffassung, dass , die Zusammenarbeit zwischen den Vereinten Nationen, den Bretton-Woods-Institutionen und der Welthandelsorganisation auch weiterhin verstärkt werden sollte, damit insbesondere die Kohärenz der Politiken und der operationellen Instrumente besser gewährleistet werden kann“19.

- Reform der Menschenrechtskommission. Bei der Menschenrechtskommission handelt es sich um eine dem ECOSOC unterstellte Fachkommission. Sie tagt in jedem Frühjahr während sechs Wochen in Genf. Die Schweiz, die der Einhaltung und Förderung des Völkerrechts besondere Bedeutung beimisst, beurteilt die derzeitige Funktionsweise der Kommission, die von zunehmender Polarisierung geprägt ist, als nicht mehr zufriedenstellend. Im Sommer 2003 beauftragte der Bund Walter Kälin, Professor an der Universität Bern, eine Studie zu diesem Thema sowie Empfehlungen zur Konkretisierung der Reform auszuarbeiten. Des Weiteren schlug Bundesrätin Micheline CalmyRey anlässlich der Eröffnung der 60. Session der UNO-Menschenrechtskommission die Schaffung eines Menschenrechtsrates vor, der - ähnlich wie der Sicherheitsrat - als quasi-permanentes Hauptorgan der Vereinten Nationen fungieren könnte.

[Dahrbuch 2005, Nr. 1, Kapitel 9, Punkt 9.5.2. „Menschliche Entwicklung und Menschenrechte“.

\subsubsection{Schweizerinnen und Schweizer bei den internationalen Organisationen}

Seit ihrem UNO-Beitritt ist es der Schweiz gelungen, ihre Präsenz in zahlreichen Organen der UNO auszubauen. Zwar scheiterten einige Kandidaturen, so etwa beim Internationalen Strafgerichtshof, aber über dreissig Schweizer Bewerbungen waren in den letzten Jahren erfolgreich ${ }^{20}$. Nachstehend werden einige Schweizer Persönlichkeiten im Dienste der Vereinten Nationen genannt: Walter Kälin, Sondervertreter des Generalsekretärs für die Menschenrechte von intern Vertriebenen (Ernennung im Oktober 2004); Nicolas Michel, Untergeneralsekretär für Rechtsangelegenheiten und Rechtsberater der UNO (Ernennung im Mai 2004); Rolf Jenny, Exekutivdirektor der Globalen Kommission für Migrationsfragen (seit 2004); Anton Thalmann, Präsident der UNO-Arbeitsgruppe gegen die illegale Verbreitung von Kleinwaffen (seit Februar 2004); Mark Pieth, Mitglied des Untersuchungsgremiums zum Hilfsprogramm „Öl für Lebensmittel“" (seit 2004); Heidi Tagliani, Leiterin der UNO-Beobachtungsmis-

19 EDA, Die Schweiz und die UNO, op. cit., S. 20.

20 Eine detaillierte Aufstellung der von Schweizerinnen und Schweizern besetzten Posten findet sich im Bericht: ibid., S. 62-63. 
sion in Georgien (seit 2002); Adolf Ogi, Sonderberater des UNO-Generalsekretärs für Sport im Dienst von Entwicklung und Frieden (seit 2001); Giorgio Malinverni, Mitglied des Ausschusses für wirtschaftliche, soziale und kulturelle Rechte (seit 2000); Jean Ziegler, UNO-Sonderberichterstatter für das Recht auf Nahrung; Carla Del Ponte, Chefanklägerin des UN-Kriegsverbrechertribunals für das ehemalige Jugoslawien (seit 1999) ${ }^{21}$; Marcel Boisard, Generaldirektor des UNITAR (seit 1972).

\subsubsection{Session der Generalversammlung der Vereinten Nationen}

Die 59. Session der UNO-Generalversammlung wurde im September 2004 am Sitz der Vereinten Nationen in New York eröffnet. Die Tagesordnung umfasste mehr als 150 Traktanden. Die Schweizer Delegation an der 59. Session der UNO-Generalversammlung setzte sich für die folgenden drei Prioritäten ein ${ }^{22}$ :

- Priorität 1: Fortsetzung der Reformbestrebungen im Hinblick auf eine Stärkung der Rolle der UNO:

- Umsetzung von Reformen,

- verstärkte Einbindung der Zivilgesellschaft und des Privatsektors,

- effiziente Verwendung der Finanzmittel der UNO.

- Priorität 2: Engagement für eine nachhaltige Entwicklung und die Folgearbeiten und Umsetzung der Millenniums-Entwicklungsziele:

- Folgearbeiten zum Millenniumsgipfel - Millennium+,

- Dreijahresprüfung der Entwicklungstätigkeit,

- soziale Dimension der Globalisierung,

- Schutz der natürlichen Ressourcen und der Umwelt,

- Unterstützung der zweiten Phase des WSIS,

- Unterstützung des Jahres des Sports im Dienste von Frieden und Entwicklung.

口 Priorität 3: Förderung der menschlichen Sicherheit:

- Friedens- und Sicherheitsförderung durch Rüstungskontrolle und Abrüstung,

- Förderung und Schutz der Menschenrechte,

- Terrorismusbekämpfung,

- Sicherheit von Personal und Einrichtungen der UNO,

- Unterstützung des Internationalen Strafgerichtshofes.

\subsection{Schweiz - Europäische Union: politische Einigung über die Bilateralen II}

Mit der Unterzeichnung der bilateralen Abkommen II zwischen der Schweiz und der Europäischen Union im Oktober 2004 in Brüssel wurde im vergangenen Jahr ein aussenpolitischer Meilenstein gesetzt. Einige Aspekte der Beziehungen zwischen der Schweiz und der EU, die in diesen Abkommen geregelt werden, stehen im Zusammenhang mit den Beziehungen der Schweiz zu den Ländern des Südens und Ostens. Sie werden in den entsprechenden Kapiteln des vorliegenden Jahrbuchs vertieft.

21 Le Temps, 19. Mai 2004.

22 EDA, Prioritäten der Schweiz an der 59. Session der Generalversammlung der Vereinten Nationen und Schweizer Delegation an der Debatte auf hoher Ebene, Pressemitteilung, 25. August 2004. 
Die als „Bilaterale II“ bezeichneten Abkommen ergänzen die bilateralen Abkommen I aus dem Jahr 1999 und bilden die Fortsetzung des bilateralen Weges, den die Schweiz nach der Ablehnung des Beitritts zum Europäischen Wirtschaftsraum (EWR) eingeschlagen hat. Die Schweiz will auf diese Weise ihre Interessen wahren und konkrete Fragestellungen in ihren Beziehungen zur Europäischen Union (EU) pragmatisch durch den Abschluss bilateraler Vereinbarungen lösen. Die Verhandlungen über die Bilateralen II betrafen neben wichtigen wirtschaftlichen Interessen (Finanzplatz, Nahrungsmittelindustrie, Tourismus) auch eine Intensivierung der Zusammenarbeit in bedeutenden Bereichen der Politik, darunter die innere Sicherheit, das Asylwesen, die Umwelt und die Kultur. Im Rahmen der Bilateralen Verhandlungen II wurden Einigungen in neun Themenbereichen erzielt, wovon acht vom Parlament gebilligt werden müssen ${ }^{23}$.

$\mathrm{Zu}$ den umstrittenen Themen zählt unter anderem die Teilnahme der Schweiz am System von Schengen/Dublin (polizeiliche Zusammenarbeit, Rechtshilfe, Asyl und Migrationspolitik), dessen Zustandekommen potenziell mit der Wahrung der Interessen des Finanzplatzes Schweiz in Konflikt stand. Die Vereinbarung von Mai 2004 garantiert, dass die Schweiz das Bankgeheimnis für direkte Steuern beibehalten kann. In der Schlussphase der Verhandlungen gab die Schweiz ihre Absicht bekannt, eine Milliarde Franken an die soziale und wirtschaftliche Kohäsion der erweiterten EU beizusteuern ${ }^{24}$.

Jahrbuch 2005, Nr. 1, Kapitel 3, „Zusammenarbeit mit Osteuropa und der GUS“; Kapitel 8, „Finanzplatz Schweiz“; Kapitel 12, „Asylpolitik und Migrationsfragen“.

\subsection{Beziehungen zwischen der Schweiz und Südafrika}

Die schweizerischen Beziehungen zum südafrikanischen Apartheidregime sorgen in den Medien, in den NGO sowie in der Öffentlichkeit seit langem für polemische Diskussionen. Die Thematik war aber auch Gegenstand zahlreicher parlamentarischer Vorstösse ${ }^{25}$, was die Delegation der Geschäftsprüfungskommission der Eidgenössischen Räte (GPDel) dazu veranlasste, mehrere Untersuchungen in die Wege zu leiten. Nach $1993^{26}$ wurde 2004 ein weiterer Bericht mit Untersuchungsergebnissen veröffentlicht (siehe unten). In der Zwischenzeit, 1999, erschien auch der Bericht der Interdepartementalen Arbeitsgruppe „Schweiz-Südafrika“" ${ }^{27}$, die bei ihrer Aufarbeitung des Verhältnisses zwischen den beiden Ländern die Beziehungen des schweizerischen Nachrichtendienstes zu südafrikanischen Stellen und Personen bewusst ausgeklammert hatte. Eine der Schlussfolgerungen dieses Berichts lautete, dass weiterführende Untersuchungen gefördert werden sollten, um die Politik der Schweiz gegenüber Südafrika aus

23 seco, Die Volkswirtschaft, Monatsthema: „Bilaterale Abkommen II“, September 2004.

24 Bundeskanzlei, Gipfeltreffen Schweiz - EU: Politische Einigung in den bilateralen Verhandlungen, Medienmitteilung, 19. Mai 2004.

25 Seit 1963 wurden bereits mehr als 150 parlamentarische Vorstösse über die Beziehungen zwischen den beiden Ländern vom Bundesrat beantwortet.

26 Der Bericht vom 28. September 1993 (BB1 1994 I 100) befasste sich mit dem Austausch von Piloten zwischen der Schweiz und Südafrika. Seinerzeit gelangte die GPDel zum Schluss, dass der Austausch von Berufsmilitärpiloten zwischen 1983 und 1988 einem militärischen Bedürfnis entsprochen und die Schweiz damit weder Neutralitätsrecht noch andere völkerrechtliche Verpflichtungen verletzt habe.

27 Dieser Bericht wurde vom Bundesrat im Oktober 1999 genehmigt. 
globaler Sicht beurteilen zu können. Als Antwort darauf erteilte der Bundesrat den zuständigen Bundesämtern den Auftrag, die Einbettung entsprechender Forschungsarbeiten in das nationale Forschungsprogramm „Grundlagen und Möglichkeiten der Schweizerischen Aussenpolitik“ (NFP 42) in die Wege zu leiten.

\subsubsection{Untersuchung über die Kontakte des Schweizer Nachrichtendienstes zu Südafrika zur Zeit des Apartheidregimes}

Der Bericht aus dem Jahr $2004^{28}$ befasst sich schwerpunktmässig mit der Rolle, die der Schweizer Nachrichtendienst in seinen Beziehungen zu Südafrika seit dem Ende der 70er-Jahre gespielt hat, sowie mit der Rolle von Divisionär Peter Regli als Chef der Untergruppe Nachrichtendienst. Während knapp zwei Jahren führte die Delegation Nachforschungen durch und schöpfte dabei alle in der Schweiz verfügbaren Informationsquellen aus. Hingegen verweigerte die Regierung unter Präsident Mbeki ihr die Erlaubnis, auch in Südafrika Nachforschungen anzustellen.

Die Delegation gelangte zum Schluss, dass sich „die Kontakte des Schweizer Nachrichtendienstes mit Südafrika auf Grund der damaligen Umstände erklären lassen. Die Kontakte waren aus neutralitäts- und aussenpolitischer Sicht sehr kritisierbar, aber in der Logik des Kalten Krieges nachvollziehbar. [...] Nach Ansicht der Delegation wäre es Aufgabe des damaligen Bundesrates gewesen, die militärische Rechtfertigung der Kontakte mit Südafrika und ihre politische Opportunität zu beurteilen. Diese Einschätzung wurde aber vorwiegend dem Nachrichtendienst überlassen, welcher die militärische Logik über diejenige der politischen Behörden stellte." ${ }^{29}$ In dem Bericht wird ferner Divisionär Peter Regli entlastet. Die Delegation habe keinen Hinweis darauf gefunden, dass Divisionär Regli oder Dienststellen des Bundes beim Aufbau des südafrikanischen Biologie- und Chemiewaffenprogramms in irgendeiner Weise beteiligt gewesen waren. Allerdings wird festgestellt, dass „Divisionär Regli zahlreichen Fragen ausgewichen ist oder bei früheren Untersuchungen Tatsachen unerwähnt

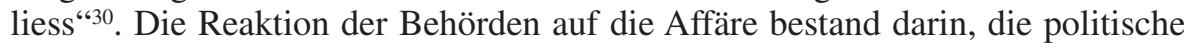
und parlamentarische Kontrolle über den Nachrichtendienst stark auszubauen. Die Aktivitäten des Nachrichtendienstes richten sich nunmehr nach den aussenpolitischen Grundsätzen des Bundesrates.

$\mathbb{1}$ Jahrbuch 2000, Kapitel 6.5. „Bericht über die Beziehungen zwischen der Schweiz und Südafrika“, S. 300-301.

\subsubsection{NFP 42+, „Beziehungen Schweiz - Südafrika“}

Im Mai 2000 wurde der Schweizerische Nationalfonds zur Förderung der wissenschaftlichen Forschung (SNF) vom Bundesrat mit der Errichtung eines Zusatzmoduls „Schweiz - Südafrika“ im Rahmen des Nationalen Forschungsprogramms „Grundlagen und Möglichkeiten der schweizerischen Aussenpolitik“

28 Untersuchung über die Kontakte des Schweizer Nachrichtendienstes zu Südafrika zur Zeit des Apartheidregimes. Bericht der Delegation der Geschäftsprüfungskommission der Eidgenössischen Räte vom 18. August 2003 (BB1 2004 2267).

29 Ibid., S. 2270.

30 Ibid., S. 2271. 
(NFP 42) beauftragt. Die über drei Jahre geplante wissenschaftliche Analyse soll die jüngere Vergangenheit der Aussenpolitik und des Aussenhandels der Schweiz mit Südafrika aufarbeiten. Für die Durchführung des Zusatzmoduls wurden zwei Millionen Franken gesprochen ${ }^{31}$.

Am 17. April 2003 liess der Bundesrat per Medienmitteilung verlauten, er habe entschieden, den Zugang zu Südafrika-Akten im Bundesarchiv, welche die Apartheidzeit betreffen und Namen von Unternehmen enthalten, vorübergehend zu unterbinden. Grund für diese Entscheidung seien die in den USA eingereichten Sammelklagen gegen in- und ausländische Firmen, die zur Zeit des Apartheidregimes geschäftliche Beziehungen zu Südafrika unterhielten. Die bisher praktizierte freie Akteneinsicht berge die Gefahr, die Stellung der eingeklagten Schweizer Firmen gegenüber mitbetroffenen ausländischen Unternehmen im Rahmen der Sammelklagen zu verschlechtern ${ }^{32}$.

Die Entscheidung der Landesregierung löste in Akademiker- und Parlamentskreisen heftige Reaktionen aus. Der Historiker Georg Kreis, Präsident der Leitungsgruppe des NFP 42+, erklärte, schon jetzt sei klar, dass das NFP 42+ kein Ruhmesblatt in der schweizerischen Forschungsgeschichte darstelle. Der Bundesrat habe seinen expliziten Willen zur Aufarbeitung der Beziehungen zwischen der Schweiz und Südafrika, den er im Frühjahr 2000 mit der Lancierung des Programms bekundete, mit seinem Entscheid vom April 2003 ins Gegenteil verkehrt $^{33}$. Am 19. Juni 2003 veranlasste der Vorfall auch Nationalrätin Pia Hollenstein zu einer Interpellation ${ }^{34}$ an den Bundesrat, die 18 Monate später noch immer nicht im Plenum erörtert worden war.

\section{QUELLEN}

EDA, Pressedossier zur Jahresmedienkonferenz 2004 des EDA, 22. April 2004.

EDA, Die Schweiz und die UNO. Bericht des Bundesrats 2004, Bern, 2004. Ebenfalls erschienen im Bundesblatt: Bericht 2004 über die Beziehungen zur UNO und zu den internationalen Organisationen mit Sitz in der Schweiz vom 7. Juni 2004 (BB1 2004 3885).

EDA, „UNO-Reform: Neuer Anlauf im Zeichen neuer Bedrohungen“, Schweiz Global, Nr. 3, 2004.

Untersuchung über die Kontakte des Schweizer Nachrichtendienstes zu Südafrika zur Zeit des Apartheidregimes. Bericht der Delegation der Geschäftsprüfungskommission der Eidgenössischen Räte vom 18. August 2003 (BB1 2004 2267).

Bundesversammlung, Amtliches Bulletin, Bern.

Bericht über die im Jahr 2003 abgeschlossenen internationalen Verträge vom 18. Mai 2004 (BB1 2004 3319).

Aussenpolitischer Bericht 2000 - Präsenz und Kooperation: Interessenwahrung in einer zusammenwachsenden Welt, 15. November 2000 (BB1 2001 261).

Bericht über die Legislaturplanung 2003-2007 vom 25. Februar 2004 (BB1 2004 1149).

seco, Die Volkswirtschaft, Monatsthema: „Bilaterale Abkommen II“, September 2004.

\section{INTERNET-ADRESSEN}

Eidgenössisches Departement für auswärtige Angelegenheiten (EDA) : <www.eda.admin.ch>.

Vereinte Nationen: <www.un.org $>$.

Schweizer Parlament: <www.parlament.ch>.

31 EDI, Wissenschaftliche Analyse der Beziehungen Schweiz-Südafrika, Pressemitteilung, 3. Mai 2000.

32 EFD, Neuregelung der Akteneinsicht betreffend Südafrika zur Apartheid-Zeit, Medienmitteilung, 17. April 2003.

33 SNF, „Schweiz-Südafrika: Abgewürgte Forschung“, Horizonte, September 2003.

34 Bundesversammlung, 03.3666 - Interpellation. Aktensperre betreffend Archivbestände Schweiz/Südafrika. Offene Fragen, eingereicht von Pia Hollenstein, Einreichungsdatum 19. Juni 2003, Amtliches Bulletin, Herbstsession 2003, 16. Sitzung, <www.parlament.ch>. 